\title{
Solution and Analysis of a One-Dimensional First-Passage Problem with a Nonzero Halting Probability
}

\author{
Ken Yamamoto \\ Department of Physics, Faculty of Science and Engineering, Chuo University, Kasuga, Bunkyo, Tokyo 112-8551, Japan \\ Correspondence should be addressed to Ken Yamamoto; yamamoto@phys.chuo-u.ac.jp
}

Received 24 April 2013; Accepted 9 September 2013

Academic Editor: Michel Droz

Copyright (C) 2013 Ken Yamamoto. This is an open access article distributed under the Creative Commons Attribution License, which permits unrestricted use, distribution, and reproduction in any medium, provided the original work is properly cited.

\begin{abstract}
This paper treats a kind of a one-dimensional first-passage problem, which seeks the probability that a random walker first hits the origin at a specified time. In addition to a usual random walk which hops either rightwards or leftwards, the present paper introduces the "halt" that the walker does not hop with a nonzero probability. The solution to the problem is expressed using a Gauss hypergeometric function. The moment generating function of the hitting time is also calculated, and a calculation technique of the moments is developed. The author derives the long-time behavior of the hitting-time distribution, which exhibits power-law behavior if the walker hops to the right and left with equal probability.
\end{abstract}

\section{Introduction}

The first-passage problem is a useful and fundamental problem in statistical physics [1]. In this problem we seek the probability that a diffusion particle or a random walker first reaches a specific set of sites at a specified time. The firstpassage problem has many applications in statistical physics, such as reaction-rate theory [2], neuron dynamics [3], and economic analysis [4].

The simplest form of the first-passage problem is a random walker hopping on a one-dimensional lattice until it hits the origin. This problem is called the gambler's ruin problem in probability theory [5] - the motion of the random walker is regarded as the dynamics of a gambler's bankroll. Consider a random walker on a one-dimensional lattice hopping to the right with probability $p_{\rightarrow}$ and left with $p_{\leftarrow}\left(=1-p_{\rightarrow}\right)$ in a single time step. The problem seeks statistical properties of duration $T_{x}$, the time at which the walker from the position $x$ first hits the origin (of course, $T_{x}$ is a random variable.) The central quantity is the probability $\widetilde{P}(x, t)$ that the walker at $x$ first hits the origin after time $t$. According to [5], the solution is obtained as

$$
\widetilde{P}(x, t)=\left\{\begin{array}{l}
\frac{x}{t}\left(\begin{array}{c}
t \\
\frac{t+x}{2}
\end{array}\right) p_{\rightarrow}^{(t-x) / 2} p_{\leftarrow}^{(t+x) / 2} \\
t \text { and } x \text { are of same parity, } \\
0 \text { and } x \text { are of the opposite parity, }
\end{array}\right.
$$

by solving the equation $\widetilde{P}(x, t+1)=p_{\rightarrow} \widetilde{P}(x+1, t)+p_{\leftarrow} \widetilde{P}(x-$ $1, t)$ with initial and boundary conditions $\widetilde{P}(0,0)=1$ and $\widetilde{P}(x, 0)=\widetilde{P}(0, t)=0(x, t \geq 1)$. The coefficient before $p_{\rightarrow}^{(t-x) / 2} p_{\leftarrow}^{(t+x) / 2}$ in (1) is the number of different paths from $x$ hitting the origin first at time $t$, and it is connected with the reflection principle of a random walk [6]. Obviously, $\widetilde{P}(x, t)=$ 0 holds when $t<x$, because $\left(\begin{array}{c}t \\ (t+x) / 2\end{array}\right)=0$.

The present paper analyzes an extended form of the above one-dimensional first-passage problem; a random walker hops to the right with probability $p_{\rightarrow}$, to the left with $p_{\hookleftarrow}$, and it does not hop with probability $p_{0}=1-p_{\rightarrow}-p_{\leftarrow}$. Figure 1 schematically shows the problem. The only difference from the original ruin problem is that the halting probability $p_{0}$ is introduced, but the results change greatly. In fact, the solution (1) of nonhalting case $\left(p_{0}=0\right)$ is superseded by a formula involving a Gauss hypergeometric function in halting case $p_{0} \neq 0$. Moreover, moment analysis and asymptotic (longtime) behavior are developed.

\section{Hypergeometric Solution}

As in the classical problem described in the previous section, let $P(x, t)$ be the probability that the walker starting from $x$ has duration $T_{x}=t . P(x, t)$ satisfies the equation $P(x, t+1)=$ $p_{\rightarrow} P(x+1, t)+p_{\leftarrow} P(x-1, t)+p_{0} P(x, t)$ with initial and boundary conditions $P(0,0)=1$ and $P(x, 0)=P(0, t)=0$ 


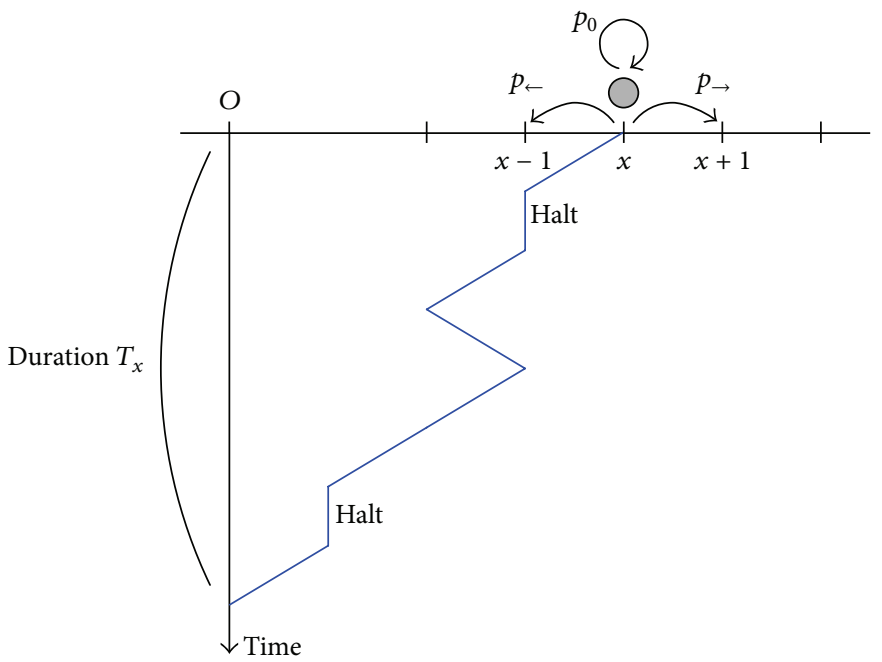

FIgURE 1: An illustration of the problem. The random walker hops to the right with probability $p_{\rightarrow}$ and to the left with $p_{\leftarrow}$; hopping does not occur with probability $p_{0}$. $\left(p_{\rightarrow}+p_{\leftarrow}+p_{0}=1\right)$. The problem focuses on statistical properties of the duration $T_{x}$, which is the time the walker first hits the origin.

$(x, t \geq 1)$. However, it is hard to solve this equation directly, and we take another way by employing a classical result (1) effectively.

To calculate $P(x, t)$, we classify the walker's paths according to the number of hopping. We count the paths consisting of $j$ hops and $t-j$ halts. First, the different patterns of putting $t-j$ halts into $t$ steps are given by $\left(\begin{array}{c}t-1 \\ t-j\end{array}\right)$ in total, where " $t-1$ " (not $t$ ) comes from the fact that a halting step never comes to the last $t$ th step. Next, if we focus on only the hopping steps (and ignore the halting steps), the paths are reduced to those of classical ruin problem; the probability that the walker from $x$ hits the origin after $j$ hops is given by $\widetilde{P}(x, j)$. Thus, the total occurring probability of a path with $j$ hops and $t-j$ halts is

$$
\left(\begin{array}{l}
t-1 \\
t-j
\end{array}\right) p_{0}^{t-j} \widetilde{P}(x, j)
$$

Summing up all $j$, we get

$$
\begin{aligned}
P(x, t) & \\
= & \sum_{j=x}^{t}\left(\begin{array}{l}
t-1 \\
t-j
\end{array}\right) p_{0}^{t-j} \widetilde{P}(x, j) \\
= & \sum_{\substack{j=x \\
j+x \text { even }}}^{t} p_{\rightarrow}^{(j-x) / 2} p_{\leftarrow}^{(j+x) / 2} p_{0}^{t-j} x \\
& \times \frac{(t-1) !}{((j+x) / 2) !((j-x) / 2) !(t-j) !} \\
= & \sum_{\substack{j=x \\
j+x \text { even }}}^{\infty} p_{\rightarrow}^{(j-x) / 2} p_{\leftarrow}^{(j+x) / 2} p_{0}^{t-j} x
\end{aligned}
$$

$$
\begin{aligned}
& \times \frac{(t-1) !}{((j+x) / 2) !((j-x) / 2) !(t-j) !} \\
= & \sum_{k=0}^{\infty} p_{\rightarrow}^{k} p_{\leftarrow}^{x+k} p_{0}^{t-x-2 k} x \frac{(t-1) !}{(x+k) ! k !(t-x-2 k) !} .
\end{aligned}
$$

In the third equality, the range of summation is changed; the terms corresponding to $j>t$ have no contribution because $1 /((t-j) !)=0$. In the last equality we change the summation variable as $j=x+2 k$. This result can be also obtained by solving directly the equation $P(x, t+1)=p_{\rightarrow} P(x+1, t)+$ $p_{\leftarrow} P(x-1, t)+p_{0} P(x, t)$ with conditions $P(0,0)=1$ and $P(x, 0)=P(0, t)=0(x \geq 1, t \geq 1)$.

We further proceed with calculation; we rewrite factorials using gamma functions, which is a preparatory step toward a hypergeometric function. Employing some formulas of the gamma function, one obtains

$$
\begin{aligned}
& \frac{1}{(t-x-2 k) !} \\
& =\frac{1}{\Gamma(t+1-x)} \\
& \quad \times \frac{\Gamma(((x-t) / 2)+(1 / 2)+k) \Gamma(((x-t) / 2)+k)}{\Gamma(((x-t) / 2)+(1 / 2)) \Gamma((x-t) / 2)} 2^{2 k} .
\end{aligned}
$$

See Appendix A for the calculation in detail. The probability $P(x, t)$ is expressed as

$$
\begin{aligned}
& P(x, t) \\
& =p_{\leftarrow}^{x} p_{0}^{t-x} x \frac{(t-1) !}{\Gamma(x+1) \Gamma(t-x+1)} \\
& \quad \times \frac{\Gamma(x+1)}{\Gamma(((x-t) / 2)+(1 / 2)) \Gamma((x-t) / 2)}
\end{aligned}
$$




$$
\begin{aligned}
& \times \sum_{k=0}^{\infty} \frac{\Gamma(((x-t) / 2)+k+(1 / 2)) \Gamma(((x-t) / 2)+k)}{\Gamma(x+k+1)} \\
& \times \frac{1}{k !}\left(\frac{4 p_{\rightarrow} p_{\leftarrow}}{p_{0}^{2}}\right)^{k} \\
& =p_{\leftarrow}^{x} p_{0}^{t-x} \frac{(t-1) !}{(x-1) !(t-x) !} \\
& \quad \times F\left(\frac{x-t}{2}, \frac{x-t+1}{2} ; x+1 ; \frac{4 p_{\rightarrow} p_{\leftarrow}}{p_{0}^{2}}\right),
\end{aligned}
$$

where $F(\alpha, \beta ; \gamma ; z)$ is the Gauss hypergeometric function. This is an explicit form of the solution of our problem. This solution cannot be deduced from the nonhalting solution (1). The hypergeometric function in (5) is a genuine hypergeometric function, in the sense that it cannot be expressed using simpler functions. (It has been studied that some hypergeometric functions have tractable expressions, e.g., $F\left(1 / 2,1 ; 3 / 2 ;-z^{2}\right)=z^{-1} \arctan z[7]$.)

We comment here on the convergence of the sum in (5)at first sight, it seems to diverge when $4 p_{\rightarrow} p_{\leftarrow} / p_{0}^{2}>1$. By using the Pochhammer symbol $(\alpha)_{k}:=\alpha(\alpha+1) \cdots(\alpha+k-1)$ instead of gamma functions,

$$
\begin{aligned}
P(x, t)= & p_{\leftarrow}^{x} p_{0}^{t-x} \frac{(t-1) !}{(x-1) !(t-x) !} \\
& \times \sum_{k=0}^{\infty} \frac{((x-t+1) / 2)_{k}((x-t) / 2)_{k}}{(x+1)_{k}} \frac{1}{k !}\left(\frac{4 p_{\rightarrow} p_{\leftarrow}}{p_{0}^{2}}\right)^{k} .
\end{aligned}
$$

If $x>t, P(x, t)=0$ holds automatically because $1 /(t-$ $x) !=0$. On the other hand, if $x \leq t$, either $((x-t) / 2)_{k}$ or $((x-t+1) / 2)_{k}$ becomes zero for $k>\lfloor(t-x) / 2\rfloor$. (More precisely, the former becomes zero when $x$ and $t$ are of same parity, and the latter becomes zero otherwise.) The sum consists of a finite number of terms in reality, so one does not need to worry about the convergence.

The exact solution (5) is difficult to understand intuitively. We show numerical evaluation of $P(x, t)$ in Figure 2 . The initial position of the walker is fixed as $x=50$. We separate graphs according to the parameter $\Delta p:=p_{\leftarrow}-p_{\rightarrow}$; Figures 2(a), 2(b), and 2(c), respectively, correspond to $\Delta p=0.2$, 0.1 , and 0 . ( $\Delta p$ is a key parameter for the average duration, as discussed in the following section.) $P(x, t)$ is a unimodal function of $t$ for each parameter value. Power-law behavior $P(x, t) \propto t^{-3 / 2}$ is suggested in large $t$ when $\Delta p=0$ (see Figure 2(d)), which is further discussed in Section 4.

\section{Analysis of Moment}

In order to see properties of the random variable $T_{x}$, moment analysis is developed in this section.

First, we calculate the moment generating function of $T_{x}$, defined as

$$
M_{x}(s)=\sum_{t=0}^{\infty} e^{s t} P(x, t)
$$

Calculation process is summarized in Appendix B, and the result is

$$
M_{x}(s)=\left(\frac{e^{-s}-p_{0}}{2 p_{\rightarrow}}-\sqrt{\left(\frac{e^{-s}-p_{0}}{2 p_{\rightarrow}}\right)^{2}-\frac{p_{\leftarrow}}{p_{\rightarrow}}}\right)^{x} .
$$

Remarkably, $M_{x}(s)$ contains no special functions, though the infinite summation in (7) involves a genuine hypergeometric function via $P(x, t)$.

Repeatedly differentiating $M_{x}(s)$ and putting $s=0$, we have the first and second moments of the duration $T_{x}$ as

$$
\begin{gathered}
\left\langle T_{x}\right\rangle=\frac{x}{p_{\leftarrow}-p_{\rightarrow}}, \\
\left\langle T_{x}^{2}\right\rangle=\frac{x^{2}}{\left(p_{\leftarrow}-p_{\rightarrow}\right)^{2}}+\left\{\frac{p_{\leftarrow}+p_{\rightarrow}}{\left(p_{\leftarrow}-p_{\rightarrow}\right)^{3}}-\frac{1}{p_{\leftarrow}-p_{\rightarrow}}\right\} x .
\end{gathered}
$$

The average and the other moments remain finite when $p_{\leftarrow}>$ $p_{\rightarrow}$, and they diverge to infinity as $p_{\leftarrow}-p_{\rightarrow} \searrow 0$. The variance of $T_{x}$ is given by

$$
V\left(T_{x}\right):=\left\langle T_{x}^{2}\right\rangle-\left\langle T_{x}\right\rangle^{2}=\left\{\frac{p_{\leftarrow}+p_{\rightarrow}}{\left(p_{\leftarrow}-p_{\rightarrow}\right)^{3}}-\frac{1}{p_{\leftarrow}-p_{\rightarrow}}\right\} x .
$$

We can give a plain explanation for the average $\left\langle T_{x}\right\rangle$. The walker moves to the left by a length $\Delta p=p_{\leftarrow}-p_{\rightarrow}$ on average in a single hop. In other words, $\Delta p$ is a mean velocity of the walker toward the origin. Hence, it takes $x / \Delta p$ time steps on average to reach the origin.

The moment generating function (8) looks too complicated to calculate higher moments by differentiation. Alternatively, we provide another calculation method for moments of the duration $T_{x}$.

We start from the difference equation for $P(x, t)$ :

$$
P(x, t+1)=p_{\rightarrow} P(x+1, t)+p_{\leftarrow} P(x-1, t)+p_{0} P(x, t) .
$$

Multiply $t$ and take summation for $t$ to get the following:

$$
\begin{array}{rl}
\sum_{t=0}^{\infty} t & P(x, t+1) \\
& =\sum_{t=0}^{\infty}\left\{t p \rightarrow P(x+1, t)+t p_{\leftarrow} P(x-1, t)+t p_{0} P(x, t)\right\} \\
& =p_{\rightarrow}\left\langle T_{x+1}\right\rangle+p_{\leftarrow}\left\langle T_{x-1}\right\rangle+p_{0}\left\langle T_{x}\right\rangle .
\end{array}
$$

The left-hand side can be expressed as

$$
\begin{aligned}
\sum_{t=0}^{\infty} t P(x, t+1)= & \sum_{t=0}^{\infty}(t+1) P(x, t+1) \\
& -\sum_{t=0}^{\infty} P(x, t+1)=\left\langle T_{x}\right\rangle-1 .
\end{aligned}
$$




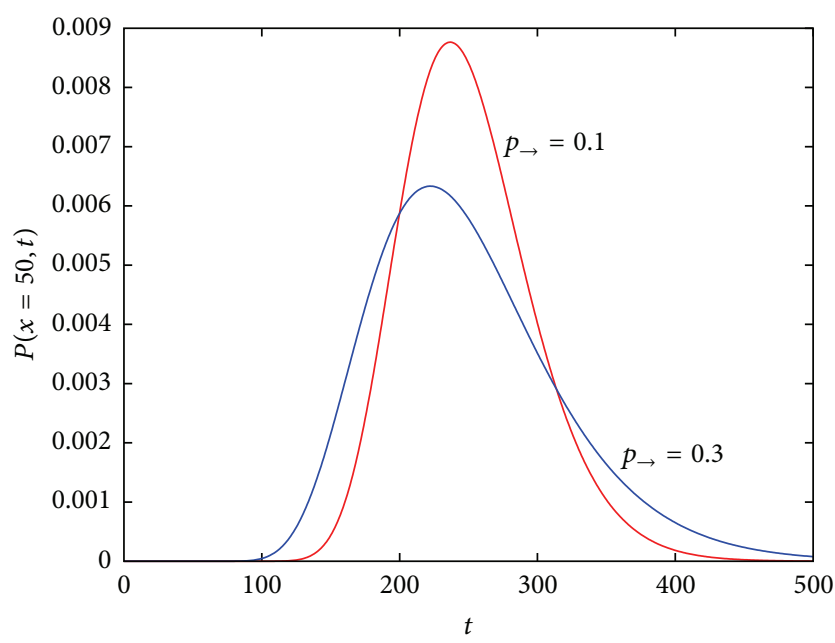

(a)

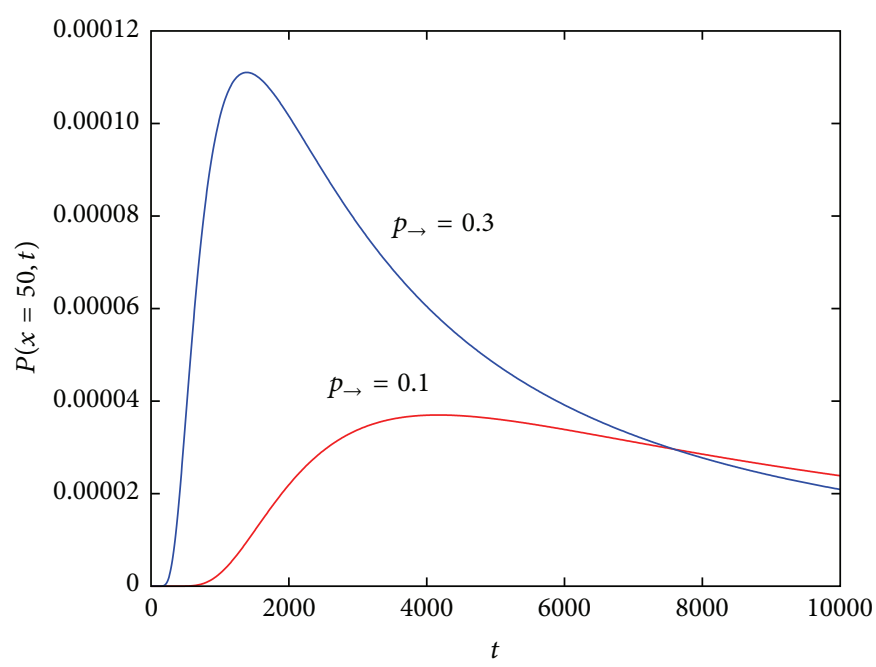

(c)

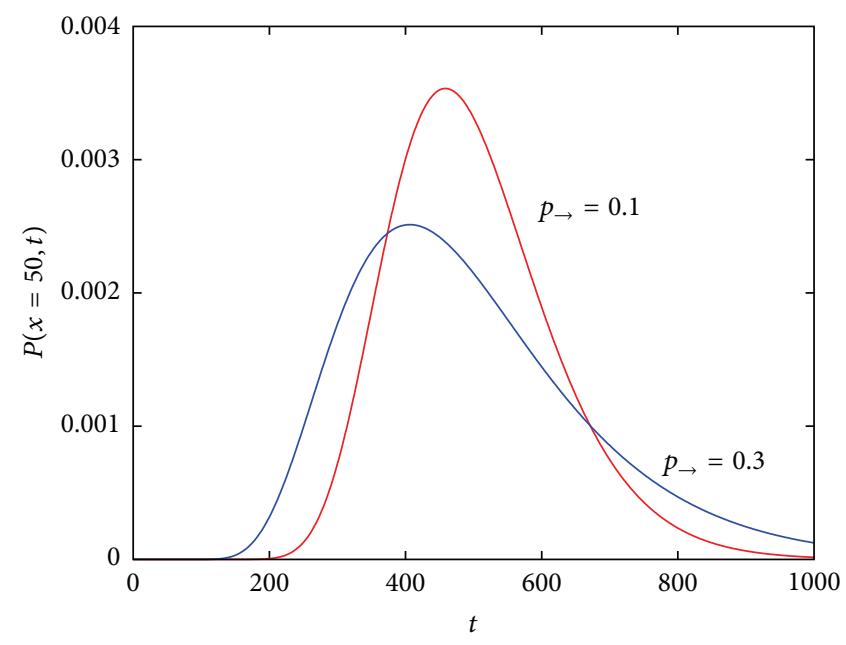

(b)

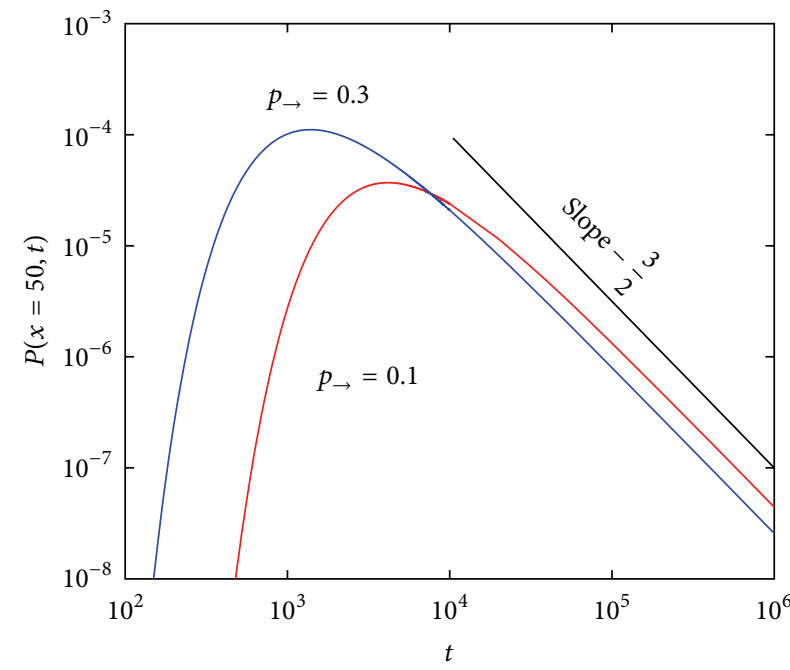

(d)

Figure 2: Numerical results of $P(x, t)$ as a function of $t(x=50)$. The graphs are separated according to the value of $\Delta p=p_{\leftarrow}-p \rightarrow$ : (a) $\Delta p=0.2$, (b) $\Delta p=0.1$, and (c) $\Delta p=0$. The two curves in each panel represent $p_{\rightarrow}=0.1$ and 0.3 . Long-time behavior of $P(x, t)$ in $\Delta p=0$ is shown in (d) on logarithmic scales, which suggests a power law $P(x, t) \propto t^{-3 / 2}$.

The last equality uses $\sum_{t=0}^{\infty} P(x, t+1)=1$, which means that a random walker surely hits the origin. (This normalization breaks if $p_{\leftarrow}<p_{\rightarrow}$ - see the discussion of total probability of hitting the origin in Section 5 in more detail.) We have a difference equation:

$$
p_{\rightarrow}\left\langle T_{x+1}\right\rangle-\left(p_{\rightarrow}+p_{\leftarrow}\right)\left\langle T_{x}\right\rangle+p_{\leftarrow}\left\langle T_{x-1}\right\rangle=-1 .
$$

A particular solution is given by $\left\langle T_{x}\right\rangle=x /\left(p_{\leftarrow}-p_{\rightarrow}\right)$, and the characteristic equation $p_{\rightarrow} \lambda^{2}-\left(p_{\rightarrow}+p_{\leftarrow}\right) \lambda+p_{\leftarrow}=0$ admits the two roots 1 and $p_{\leftarrow} / p_{\rightarrow}$. Thus, the general solution is

$$
\left\langle T_{x}\right\rangle=\frac{x}{p_{\leftarrow}-p_{\rightarrow}}+A+B\left(\frac{p_{\leftarrow}}{p_{\rightarrow}}\right)^{x},
$$

which has two constants $A$ and $B$. In a limiting case $p_{\rightarrow} \rightarrow 0$, the problem is still well-defined, and $\left\langle T_{x}\right\rangle$ should be finite, so
$B=0$. Also, it is obvious that $\left\langle T_{0}\right\rangle=0$, so $A=0$. The appropriate solution is therefore

$$
\left\langle T_{x}\right\rangle=\frac{x}{p_{\leftarrow}-p_{\rightarrow}} .
$$

Next we calculate the second moment. Multiplying $t^{2}$ and taking summation, we derive a difference equation:

$$
\left\langle T_{x}^{2}\right\rangle-2\left\langle T_{x}\right\rangle+1=p_{\rightarrow}\left\langle T_{x+1}^{2}\right\rangle+p_{\leftarrow}\left\langle T_{x-1}^{2}\right\rangle+p_{0}\left\langle T_{x}^{2}\right\rangle,
$$

where we use $t^{2}=(t+1)^{2}-2(t+1)+1$ for the calculation on the left-hand side. We assume a particular solution in the form $\left\langle T_{x}^{2}\right\rangle=C_{2} x^{2}+C_{1} x$, and determine two constants as

$$
\begin{gathered}
C_{1}=\frac{p_{\leftarrow}+p_{\rightarrow}}{\left(p_{\leftarrow}-p_{\rightarrow}\right)^{3}}-\frac{1}{p_{\leftarrow}-p_{\rightarrow}}, \\
C_{2}=\frac{1}{\left(p_{\leftarrow}-p_{\rightarrow}\right)^{2}} .
\end{gathered}
$$


The second moment is

$$
\begin{aligned}
\left\langle T_{x}^{2}\right\rangle & =\frac{x^{2}}{\left(p_{\leftarrow}-p_{\rightarrow}\right)^{2}}+\left\{\frac{p_{\leftarrow}+p_{\rightarrow}}{\left(p_{\leftarrow}-p_{\rightarrow}\right)^{3}}-\frac{1}{p_{\leftarrow}-p_{\rightarrow}}\right\} x \\
& =\frac{x^{2}}{\left(p_{\leftarrow}-p_{\rightarrow}\right)^{2}}+\frac{p_{0}\left(1-p_{0}\right)+4 p_{\rightarrow} p_{\leftarrow}}{\left(p_{\leftarrow}-p_{\rightarrow}\right)^{3}} x .
\end{aligned}
$$

The homogeneous-solution part vanishes for the same reason as the average.

In short, this "bottom-up" method calculates higher moments inductively and generally $\left\langle T_{x}^{k}\right\rangle$ becomes a polynomial of degree $k$. For instance, the third moment is calculated as

$$
\begin{aligned}
&\left\langle T_{x}^{3}\right\rangle= \frac{x^{3}}{\left(p_{\leftarrow}-p_{\rightarrow}\right)^{3}}+\left\{\frac{p_{\leftarrow}+p_{\rightarrow}}{\left(p_{\leftarrow}-p_{\rightarrow}\right)^{3}}-\frac{1}{p_{\leftarrow}-p_{\rightarrow}}\right\} 3 x^{2} \\
&+\left\{\frac{2\left(p_{\leftarrow}+p_{\rightarrow}\right)^{2}+4 p_{\leftarrow} p_{\rightarrow}}{\left(p_{\leftarrow}-p_{\rightarrow}\right)^{5}}-\frac{3\left(p_{\leftarrow}+p_{\rightarrow}\right)}{\left(p_{\leftarrow}-p_{\rightarrow}\right)^{3}}\right. \\
&\left.+\frac{1}{p_{\leftarrow}-p_{\rightarrow}}\right\} x .
\end{aligned}
$$

\section{Asymptotic Behavior}

We look at long-time behavior of $P(x, t)$ in this section. In particular, we confirm the power-law behavior $P(x, t) \propto t^{-3 / 2}$ mentioned in Section 2 when $p_{\rightarrow}=p_{\leftarrow}$ (see Figure 2(d) for reference).

A summation and factorial, as well as a hypergeometric function, are not suitable to study limiting behavior, so we first reexpress the probability $P(x, t)$ into a tractable form (it may be also possible to estimate factorials directly by Stirling's formula). According to [5], the solution (1) of the classic ruin problem has another expression:

$$
\widetilde{P}(x, t)=2^{t} p_{\rightarrow}^{(t-x) / 2} p_{\leftarrow}^{(t+x) / 2} \int_{0}^{1} \cos ^{t-1} \pi \phi \cdot \sin \pi \phi \cdot \sin \pi x \phi d \phi .
$$

This form is convenient in that one does not need to care about the parities of $x$ and $t$. In fact, the integral holds the information of parities:

$$
\begin{aligned}
& \int_{0}^{1} \cos ^{t-1} \pi \phi \cdot \sin \pi \phi \cdot \sin \pi x \phi d \phi \\
& \quad=\left\{\begin{array}{l}
\frac{x}{2^{t}} \frac{(t-1) !}{((t+x) / 2) !((t-x) / 2) !} \\
t \text { and } x \text { are of same parity } \\
0 \\
\text { otherwise. }
\end{array}\right.
\end{aligned}
$$

In Appendix C, we prove this formula. Applying (21) to (3), we have the integral form of the probability $P(x, t)$ :

$$
\begin{aligned}
& P(x, t) \\
& =\sum_{j=1}^{t}\left(\begin{array}{l}
t-1 \\
t-j
\end{array}\right) p_{0}^{t-j} \widetilde{P}(x, j) \\
& =2 p_{\rightarrow}^{(1-x) / 2} p_{\leftarrow}^{(1+x) / 2} \\
& \times \int_{0}^{1}\left\{\sum_{j=1}^{t}\left(\begin{array}{l}
t-1 \\
t-j
\end{array}\right) p_{0}^{t-j}\right. \\
& \left.\times\left(2 \sqrt{p_{\rightarrow} p_{\leftarrow}} \cos \pi \phi\right)^{j-1}\right\} \sin \pi \phi \cdot \sin \pi x \phi d \phi \\
& =2 p_{\rightarrow}^{(1-x) / 2} p_{\leftarrow}^{(1+x) / 2} \int_{0}^{1}\left(p_{0}+2 \sqrt{p_{\rightarrow} p_{\leftarrow}} \cos \pi \phi\right)^{t-1} \\
& \times \sin \pi \phi \cdot \sin \pi x \phi d \phi \\
& =2 p_{\rightarrow}^{(1-x) / 2} p_{\leftarrow}^{(1+x) / 2}\left(p_{0}+2 \sqrt{p_{\rightarrow} p_{\leftarrow}}\right)^{t-1} \\
& \times \int_{0}^{1}\left(\frac{p_{0}+2 \sqrt{p_{\rightarrow} p_{\leftarrow}} \cos \pi \phi}{p_{0}+2 \sqrt{p_{\rightarrow} p_{\leftarrow}}}\right)^{t-1} \sin \pi \phi \cdot \sin \pi x \phi d \phi .
\end{aligned}
$$

Here we estimate the integral

$$
\begin{aligned}
I & :=\int_{0}^{1}\left(\frac{p_{0}+2 \sqrt{p_{\rightarrow} p_{\leftarrow}} \cos \pi \phi}{p_{0}+2 \sqrt{p_{\rightarrow} p_{\leftarrow}}}\right)^{t-1} \sin \pi \phi \cdot \sin \pi x \phi d \phi \\
& =\int_{0}^{\pi t}\left(\frac{p_{0}+2 \sqrt{p_{\rightarrow} p_{\leftarrow}} \cos (\nu / t)}{p_{0}+2 \sqrt{p_{\rightarrow} p_{\leftarrow}}}\right)^{t-1} \sin \frac{\nu}{t} \cdot \sin \frac{x \nu}{t} \cdot \frac{1}{\pi t} d \nu,
\end{aligned}
$$

where the integral variable is changed as $v=\pi t \phi$ in the second equality. For large $t$, consider the following:

$$
\begin{aligned}
& \left(\frac{p_{0}+2 \sqrt{p_{\rightarrow} p_{\leftarrow}} \cos (\nu / t)}{p_{0}+2 \sqrt{p_{\rightarrow} p_{\leftarrow}}}\right)^{t-1} \\
& \simeq\left(1-\frac{\sqrt{p_{\rightarrow} p_{\leftarrow}}}{p_{0}+2 \sqrt{p_{\rightarrow} p_{\leftarrow}}} \frac{\nu^{2}}{t^{2}}\right)^{t-1} \\
& \simeq \exp \left(-\frac{\sqrt{p_{\rightarrow} p_{\leftarrow}}}{p_{0}+2 \sqrt{p_{\rightarrow} p_{\leftarrow}}} \frac{\nu^{2}}{t}\right) .
\end{aligned}
$$

Hence, the integrand is a rapidly decreasing function of $v$, and we can extend the upper limit of the integration to infinity. Together with the approximation $\sin (\nu / t) \simeq v / t$, we can carry out the integration as

$$
\begin{aligned}
I & \simeq \frac{x}{\pi t^{3}} \int_{0}^{\infty} \exp \left(-\frac{\sqrt{p_{\rightarrow} p_{\leftarrow}}}{p_{0}+2 \sqrt{p_{\rightarrow} p_{\leftarrow}}} \frac{v^{2}}{t}\right) \nu^{2} d \nu \\
& =\frac{x}{4 \sqrt{\pi}}\left(\frac{p_{0}+2 \sqrt{p_{\rightarrow} p_{\leftarrow}}}{\sqrt{p_{\rightarrow} p_{\leftarrow}}}\right)^{3 / 2} t^{-3 / 2} .
\end{aligned}
$$


Therefore, the asymptotic form is expressed as

$$
\begin{aligned}
P(x, t) \simeq & \frac{x}{2 \sqrt{\pi}} p_{\rightarrow}^{(1-x) / 2} p_{\leftarrow}^{(1+x) / 2}\left(p_{0}+2 \sqrt{p_{\rightarrow} p_{\leftarrow}}\right)^{t-1} \\
& \times\left(\frac{p_{0}+2 \sqrt{p_{\rightarrow} p_{\leftarrow}}}{\sqrt{p_{\rightarrow} p_{\leftarrow}}}\right)^{3 / 2} t^{-3 / 2} .
\end{aligned}
$$

By the arithmetic mean-geometric mean inequality, we note that

$$
p_{0}+2 \sqrt{p_{\rightarrow} p_{\leftarrow}} \leq p_{0}+p_{\rightarrow}+p_{\leftarrow}=1,
$$

with equality holding if and only if $p_{\rightarrow}=p_{\leftarrow}$ (or $\Delta p=0$ by the notation in Section 2). Thus, the long-time behavior of $P(x, t)$ is completely different according to whether $\Delta p$ equals zero, or not as follows.

(i) If $\Delta p \neq 0$ (i.e., $\left.p_{\rightarrow} \neq p_{\leftarrow}\right), p_{0}+2 \sqrt{p_{\rightarrow} p_{\leftarrow}}<1$, and hence $P(x, t)$ asymptotically exhibits exponential decay due to $\left(p_{0}+2 \sqrt{p_{\rightarrow} p_{\leftarrow}}\right)^{t}$.

(ii) If $\Delta p=0$ (i.e., $p_{\rightarrow}=p_{\leftarrow}=: p$ ), a power law with exponent $-3 / 2$ is concluded:

$$
P(x, t) \simeq \frac{x}{2 \sqrt{\pi p}} t^{-3 / 2}
$$

See Figure 2(d) for reference.

\section{Concluding Remarks and Discussions}

In the present paper, we have solved a one-dimensional firstpassage problem where a random walker hops to the right with probability $p_{\rightarrow}$, left with $p_{\leftarrow}$, and does not hop with $p_{0}$. We have calculated exactly the probability $P(x, t)$ that the walker starting from position $x$ has duration $T_{x}=t$. The average and higher moments of $T_{x}$ are calculated in two ways: by the moment generating function, and by the bottom-up calculation. The asymptotic form of $P(x, t)$ is derived, and a power law $P(x, t) \sim t^{-3 / 2}$ is obtained when $p_{\rightarrow}=p_{\leftarrow}$.

We make a brief discussion on the continuum limit, which is an appropriate scaling limit where the step width and time interval of the walker tend to zero. Let the step width be $\delta$ and time interval be $\epsilon$, and position and time are scaled as $\xi=\delta x$ and $\tau=\epsilon t$. In the continuum limit we take that $\delta, \epsilon \rightarrow 0$, together with

$$
\left(p_{\rightarrow}-p_{\leftarrow}\right) \frac{\delta}{\epsilon} \longrightarrow v, \quad \frac{\delta^{2}}{2 \epsilon} \longrightarrow D,
$$

are kept finite. $v$ is the mean displacement per time unit, and $D$ is the diffusion coefficient corresponding to a nonhalting (or formally called a simple) random walk. The probability density function

$$
\rho(\xi, \tau)=\lim \frac{1}{\epsilon} P\left(\frac{x}{\delta}, \frac{t}{\epsilon}\right)
$$

is suitable in the continuum limit, rather than the probability distribution $P(x, t)$. ("lim" represents the continuum limit.) Carrying out calculation similar to that in [5],

$$
\rho(\xi, \tau)=\frac{\xi}{\sqrt{4 \pi\left(1-p_{0}\right) D \tau^{3}}} \exp \left(-\frac{(\xi+v \tau)^{2}}{4\left(1-p_{0}\right) D \tau}\right)
$$

is obtained. The probability density $\rho$ is called the inverse Gaussian distribution [8]. In the continuum limit, the halting effect $p_{0}$ is reflected only upon the diffusion coefficient as (1$\left.p_{0}\right) D$. Comparing $P(x, t)$ of a discrete problem in (5) and above $\rho(\xi, \tau)$ of a continuum limit, we conclude that the discrete random walk is far more difficult than the continuous diffusion and that the results about the discrete random walk in this paper cannot be attained from continuous diffusion problem.

Combining (5), (7), and (8), we get

$$
\begin{gathered}
\sum_{t=0}^{\infty} e^{s t} p_{\leftarrow}^{x} p_{0}^{t-x}\left(\begin{array}{c}
t-1 \\
x-1
\end{array}\right) F\left(\frac{x-t}{2}, \frac{x-t+1}{2} ; x+1 ; \frac{4 p_{\rightarrow} p_{\leftarrow}}{p_{0}^{2}}\right) \\
=\left(\frac{e^{-s}-p_{0}}{2 p_{\rightarrow}}-\sqrt{\left(\frac{e^{-s}-p_{0}}{2 p_{\rightarrow}}\right)^{2}-\frac{p_{\leftarrow}}{p_{\rightarrow}}}\right)^{x} .
\end{gathered}
$$

We have not used the condition $p_{\rightarrow}+p_{\leftarrow}+p_{0}=1$ in the derivation of (5) and (8), so $p_{\rightarrow}, p_{\leftarrow}$, and $p_{0}$ can take any value independently. Setting $p_{\rightarrow}=z / 4$ and $p_{\leftarrow}=p_{0}=1$,

$$
\begin{gathered}
\sum_{t=0}^{\infty} e^{s t}\left(\begin{array}{c}
t-1 \\
x-1
\end{array}\right) F\left(\frac{x-t}{2}, \frac{x-t+1}{2} ; x+1 ; z\right) \\
\quad=\left(\frac{2\left(e^{-s}-1\right)}{z}-\sqrt{\left.\left(\frac{2\left(e^{-s}-1\right)}{z}\right)^{2}-\frac{4}{z}\right)^{x} .}\right.
\end{gathered}
$$

Furthermore, setting $s=-\ln 2$ (i.e., $e^{-s}=2$ ),

$$
\begin{aligned}
\sum_{t=0}^{\infty} \frac{1}{2^{t}}\left(\begin{array}{c}
t-1 \\
x-1
\end{array}\right) F\left(\frac{x-t}{2}, \frac{x-t+1}{2} ; x+1 ; z\right) \\
=\left(\frac{2-2 \sqrt{1-z}}{z}\right)^{x} .
\end{aligned}
$$

These formulas are nontrivial, but the author cannot tell whether they are useful in practice. We stress that an infinite series of which each term involves a genuine hypergeometric function is hardly known.

We comment on the total probability of hitting the origin. For the discrete problem, putting $s=0$ the moment generating function $M_{x}(s)$,

$$
\begin{aligned}
& \sum_{t=0}^{\infty} P(x, t)=M_{x}(0) \\
& =\left(\frac{1-p_{0}}{2 p_{\rightarrow}}-\sqrt{\left(\frac{1-p_{0}}{2 p_{\rightarrow}}\right)^{2}-\frac{p_{\leftarrow}}{p_{\rightarrow}}}\right)^{x} \\
& =\left(\frac{p_{\rightarrow}+p_{\leftarrow}}{2 p_{\rightarrow}}-\frac{\sqrt{\left(p_{\leftarrow}-p_{\rightarrow}\right)^{2}}}{2 p_{\rightarrow}}\right)^{x} \\
& = \begin{cases}1 & p_{\leftarrow} \geq p_{\rightarrow}, \\
\left(\frac{p_{\leftarrow}}{p_{\rightarrow}}\right)^{x} & p_{\leftarrow}<p_{\rightarrow} .\end{cases}
\end{aligned}
$$


If $p_{\leftarrow}>p_{\rightarrow}$, the walker surely comes to the origin eventually, but if $p_{\leftarrow}<p_{\rightarrow}$, the walker manages to avoid hitting the origin with a nonzero probability.

The equation $P(x, t+1)=p_{\rightarrow} P(x+1, t)+p_{\leftarrow} P(x-1, t)+$ $p_{0} P(x, t)$ describes a traffic jam, where $P(x, t)$ stands for the probability distribution that the jam consists of $x$ cars at time $t$, and $p_{\rightarrow}$ and $p_{\leftarrow}$ are involved in the rates at which cars enter and leave the jam. The scaling behavior with exponent $-3 / 2$ has been observed in the lifetime distribution of jams [9]. We think that results obtained in this paper can become theoretical bases; in particular, our discrete problem is comparable to a microscopic description of traffic, where discreteness is not negligible.

\section{Appendices}

\section{A. Calculation of (4)}

Here we follow the calculation of (4) in detail. Employing the duplication formula $\Gamma(2 z)=\pi^{-1 / 2} 2^{2 z-1} \Gamma(z) \Gamma(z+(1 / 2))$, we rewrite as follows:

$$
\begin{aligned}
& \frac{1}{(t-x-2 k) !} \\
& =\frac{1}{\Gamma(t-x-2 k+1)} \\
& =\frac{\sqrt{\pi}}{2^{t-x-2 k} \Gamma(((t-x) / 2)-k+(1 / 2)) \Gamma(((t-x) / 2)-k+1)} .
\end{aligned}
$$

By Euler's reflection formula $\Gamma(z) \Gamma(1-z)=\pi / \sin \pi z$,

$$
\begin{aligned}
\Gamma\left(\frac{t-x}{2}-k+\frac{1}{2}\right) \\
=\frac{1}{\Gamma(k-((t-x) / 2)+(1 / 2))} \\
\quad \times \frac{\pi}{\sin \pi(((t-x) / 2)-k+(1 / 2))} \\
=\frac{1}{\Gamma(k-((t-x) / 2)+(1 / 2))} \\
=\frac{(-1)^{k} \pi}{\sin \pi(((t-x) / 2)+(1 / 2))} \\
\Gamma(k-((t-x) / 2)+(1 / 2)) \\
\quad \times \Gamma\left(\frac{t-x}{2}+\frac{1}{2}\right) \Gamma\left(\frac{1}{2}-\frac{t-x}{2}\right) .
\end{aligned}
$$

Note that $\sin \pi(((t-x) / 2)-k+(1 / 2))=(-1)^{k} \sin \pi(((t-$ $x) / 2)+(1 / 2))$ because $k$ is an integer. Similarly,

$$
\begin{aligned}
\Gamma\left(\frac{t-x}{2}-k+1\right)= & \frac{(-1)^{k}}{\Gamma(k+((x-t) / 2))} \\
& \times \Gamma\left(\frac{t-x}{2}+1\right) \Gamma\left(\frac{x-t}{2}\right)
\end{aligned}
$$

is obtained. Substituting (A.2) and (A.3) into (A.1), then using the duplication formula again as

$$
\Gamma\left(\frac{t-x}{2}+\frac{1}{2}\right) \Gamma\left(\frac{t-x}{2}+1\right)=\frac{\sqrt{\pi}}{2^{t-x}} \Gamma(t-x+1),
$$

we eventually come to the following result:

$$
\begin{aligned}
& \frac{1}{(t-x-2 k) !} \\
& =\frac{1}{\Gamma(t+1-x)} \\
& \quad \times \frac{\Gamma(((x-t) / 2)+(1 / 2)+k) \Gamma(((x-t) / 2)+k)}{\Gamma(((x-t) / 2)+(1 / 2)) \Gamma((x-t) / 2)} 2^{2 k} .
\end{aligned}
$$

\section{B. Calculation of the Moment Generating Function (8)}

We follow here the calculation of (8). Substituting (3) into (7),

$$
\begin{aligned}
M_{x}(s)= & \sum_{t=0}^{\infty} e^{s t} \sum_{k=0}^{\infty} p_{\rightarrow}^{k} p_{\leftarrow}^{x+k} p_{0}^{t-x-2 k} x \frac{(t-1) !}{(x+k) ! k !(t-x-2 k) !} \\
= & \sum_{k=0}^{\infty} p_{\rightarrow}^{k} p_{\leftarrow}^{x+k} p_{0}^{-x-2 k} x \frac{1}{(x+k) ! k !} \\
& \times \sum_{t=0}^{\infty} \frac{(t-1) !}{(t-x-2 k) !}\left(p_{0} e^{s}\right)^{t} \\
= & \sum_{k=0}^{\infty} p_{\rightarrow}^{k} p_{\leftarrow}^{x+k} p_{0}^{-x-2 k} x \frac{1}{(x+k) ! k !}\left(p_{0} e^{s}\right)^{x+2 k} \\
& \times \sum_{\tau=0}^{\infty} \frac{(\tau+x+2 k-1) !}{\tau !}\left(p_{0} e^{s}\right)^{\tau}
\end{aligned}
$$

where a summation variable is changed as $\tau=t-x-2 k$ in the last equality. Applying the negative binomial expansion

$$
(1-y)^{-m}=\sum_{\tau=0}^{\infty} \frac{(\tau+m-1) !}{\tau !(m-1) !} y^{\tau}
$$

with $y=p_{0} e^{s}$ and $m=x+2 k$, one obtains

$$
\sum_{\tau=0}^{\infty} \frac{(\tau+x+2 k-1) !}{\tau !}\left(p_{0} e^{s}\right)^{\tau}=(x+2 k-1) !\left(1-p_{0} e^{s}\right)^{-x-2 k}
$$

and simplifies (B.1) into

$$
M_{x}(s)=\left(\frac{p_{\leftarrow}}{e^{-s}-p_{0}}\right)^{x} x \sum_{k=0}^{\infty} \frac{(x+2 k-1) !}{(x+k) ! k !}\left(\frac{\sqrt{p_{\rightarrow} p_{\leftarrow}}}{e^{-s}-p_{0}}\right)^{2 k} .
$$


We can transform as follows using the duplication formula $\Gamma(2 z)=\pi^{-1 / 2} 2^{2 z-1} \Gamma(z) \Gamma(z+(1 / 2))$ :

$$
\begin{aligned}
(x+2 k-1) != & \Gamma(x+2 k) \\
= & \frac{2^{x-1+2 k}}{\sqrt{\pi}} \Gamma\left(\frac{x}{2}+k\right) \Gamma\left(\frac{x+1}{2}+k\right) \\
= & \frac{2^{x-1}}{\sqrt{\pi}} \Gamma\left(\frac{x}{2}\right) \Gamma\left(\frac{x+1}{2}\right) \\
& \times \frac{\Gamma((x / 2)+k) \Gamma(((x+1) / 2)+k)}{\Gamma(x / 2) \Gamma((x+1) / 2)} 2^{2 k} \\
= & \Gamma(x) \frac{\Gamma((x / 2)+k) \Gamma(((x+1) / 2)+k)}{\Gamma(x / 2) \Gamma((x+1) / 2)} 2^{2 k} .
\end{aligned}
$$

The moment generating function can be expressed by a Gauss hypergeometric function as

$$
\begin{aligned}
M_{x}(s)= & \left(\frac{p_{\leftarrow}}{e^{-s}-p_{0}}\right)^{x} \frac{x \Gamma(x)}{\Gamma(x+1)} \frac{\Gamma(x+1)}{\Gamma(x / 2) \Gamma((x+1) / 2)} \\
& \times \sum_{k=0}^{\infty} \frac{\Gamma((x / 2)+k) \Gamma(((x+1) / 2)+k)}{\Gamma(x+1+k)} \\
& \times \frac{1}{k !}\left(\frac{2 \sqrt{p_{\rightarrow} p_{\leftarrow}}}{e^{-s}-p_{0}}\right)^{2 k} \\
= & \left(\frac{p_{\leftarrow}}{e^{-s}-p_{0}}\right)^{x} F\left(\frac{x}{2}, \frac{x+1}{2} ; x+1 ; \frac{4 p_{\rightarrow} p_{\leftarrow}}{\left(e^{-s}-p_{0}\right)^{2}}\right) .
\end{aligned}
$$

It can be expressed by an elementary function as follows (see [7]):

$$
\begin{aligned}
F\left(\frac{x}{2}, \frac{x+1}{2} ; x+1 ; z\right) & =\left(\frac{2}{1+\sqrt{1-z}}\right)^{x} \\
& =\left(\frac{2-2 \sqrt{1-z}}{z}\right)^{x} .
\end{aligned}
$$

This leads to the conclusion.

\section{Proof of (22)}

We calculate the integral to prove (22). We first break up the product of circular functions into the sum:

$$
\cos ^{t-1} \pi \phi=\left(\frac{e^{i \pi \phi}+e^{-i \pi \phi}}{2}\right)^{t-1}=\frac{1}{2^{t-1}} \sum_{k=0}^{t-1}\left(\begin{array}{c}
t-1 \\
k
\end{array}\right) e^{i(2 k-t+1) \pi \phi},
$$

whose real part is

$$
\cos ^{t-1} \pi \phi=\frac{1}{2^{t-1}} \sum_{k=0}^{t-1}\left(\begin{array}{c}
t-1 \\
k
\end{array}\right) \cos (2 k-t+1) \pi \phi
$$

This is just a Fourier series expansion of $\cos ^{t-1} \theta$ and is associated with the Chebyshev polynomial $T_{n}(\cos \theta):=\cos n \theta[10]$. Moreover, by formulas in trigonometry,

$$
\begin{aligned}
& \cos (2 k-t+1) \pi \phi \cdot \sin \pi \phi \cdot \sin \pi x \phi \\
& =\frac{1}{4}\{\cos (2 k-t+x) \pi \phi+\cos (2 k+2-t-x) \pi \phi \\
& \quad-\cos (2 k+2-t+x) \pi \phi-\cos (2 k-t-x) \pi \phi\} .
\end{aligned}
$$

Thus,

$$
\begin{aligned}
& \int_{0}^{1} \cos ^{t-1} \pi \phi \cdot \sin \pi \phi \cdot \sin \pi x \phi d \phi \\
& =\frac{1}{2^{t+1}} \sum_{k=0}^{t-1}\left(\begin{array}{c}
t-1 \\
k
\end{array}\right) \\
& \times \int_{0}^{1}\{\cos (2 k-t+x) \pi \phi+\cos (2 k+2-t-x) \pi \phi \\
& \quad-\cos (2 k+2-t+x) \pi \phi \\
& \quad-\cos (2 k-t-x) \pi \phi\} d \phi .
\end{aligned}
$$

Since $t, x$, and $k$ are integers, the first term in the integral is

$$
\begin{gathered}
\int_{0}^{1} \cos (2 k-t+x) \pi \phi d \phi \\
= \begin{cases}1 & 2 k-t+x=0, \\
0 & \text { otherwise }\end{cases}
\end{gathered}
$$

which behaves like the Kronecker delta $\delta(k,(t-x) / 2)-\mathrm{a}$ subscript fraction may be unreadable, so we use $\delta(i, j)$ instead of the standard symbol $\delta_{i, j}$. The following terms in the integral are also substituted by $\delta(k,(t+x-2) / 2), \delta(k,(t-x-2) / 2)$, and $\delta(k,(t+x) / 2)$, respectively.

If $t$ and $x$ have opposite parity (i.e., $t+x$ and $t-x$ are odd), each Kronecker delta vanishes for all integer $k$. Otherwise, if $t$ and $x$ have the same parity, each Kronecker delta becomes nonzero at some $k$. By picking out such $k$,

$$
\begin{gathered}
\int_{0}^{1} \cos ^{t-1} \pi \phi \cdot \sin \pi \phi \cdot \sin \pi x \phi d \phi \\
=\frac{1}{2^{t+1}}\left\{\left(\begin{array}{c}
t-1 \\
\frac{t-x}{2}
\end{array}\right)+\left(\begin{array}{c}
t-1 \\
\frac{t+x-2}{2}
\end{array}\right)\right. \\
\left.\quad-\left(\begin{array}{c}
t-x-2 \\
\frac{t-x}{2}
\end{array}\right)-\left(\begin{array}{c}
t-1 \\
\frac{t+x}{2}
\end{array}\right)\right\} \\
=\frac{x}{2^{t}} \frac{(t-1) !}{((t+x) / 2) !((t-x) / 2) !} .
\end{gathered}
$$

Therefore, (22) is derived. 


\section{Acknowledgments}

The author is grateful to Dr. Yoshihiro Yamazaki and Dr. Junichi Wakita for their beneficial comments.

\section{References}

[1] S. Redner, A Guide to First-Passage Processes, Cambridge University Press, Cambridge, UK, 2001.

[2] P. Hänggi, "Reaction-rate theory: fifty years after Kramers," Reviews of Modern Physics, vol. 62, no. 2, pp. 251-341, 1990.

[3] T. Verechtchaguina, I. M. Sokolov, and L. Schimansky-Geier, "First passage time densities in resonate-and-fire models," Physical Review E, vol. 73, no. 3, Article ID 031108, 2006.

[4] N. Sazuka, J.-I. Inoue, and E. Scalas, "The distribution of firstpassage times and durations in FOREX and future markets," Physica A, vol. 388, no. 14, pp. 2839-2853, 2009.

[5] W. Feller, An Introduction to Probability Theory and Its Applications, vol. 1, Wiley, New York, NY, USA, 1957.

[6] D. Stanton and D. White, Constructive Combinatorics, Springer, New York, NY, USA, 1986.

[7] M. Abramowitz and I. A. Stegun, Handbook of Mathematical Functions, Dover, New York, NY, USA, 1970.

[8] M. C. K. Tweedie, "Statistical properties of inverse Gaussian distributions," Annals of Mathematical Statistics, vol. 28, pp. 362377, 1957.

[9] K. Nagel and M. Paczuski, "Emergent traffic jams," Physical Review E, vol. 51, no. 4, pp. 2909-2918, 1995.

[10] A. Gil, J. Segura, and N. M. Temme, Numerical Methods for Special Functions, SIAM, Philadelphia, Pa, USA, 2007. 

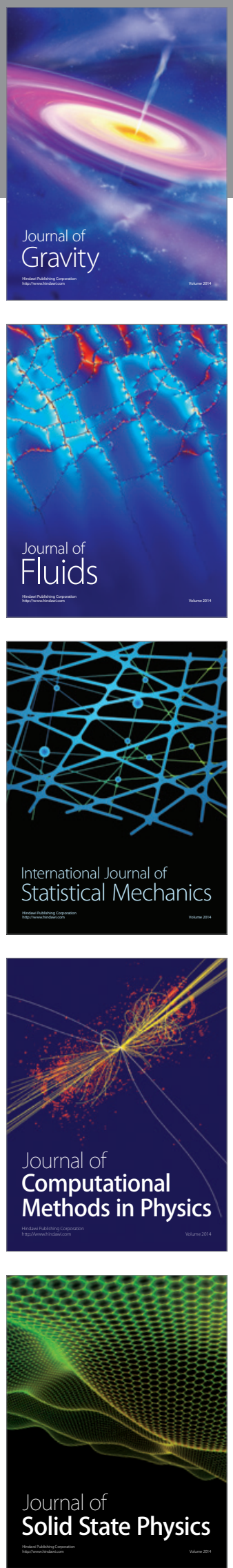

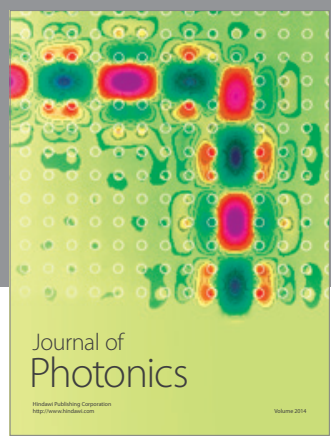

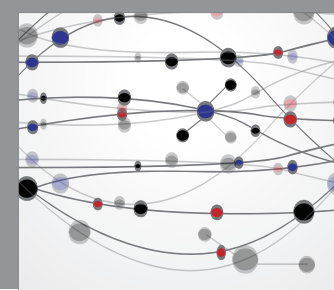

The Scientific World Journal

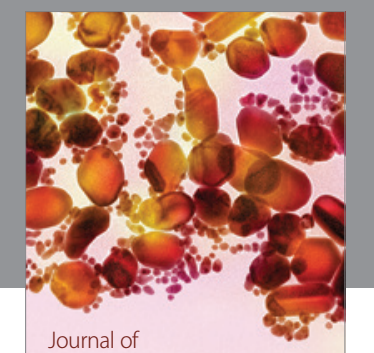

Soft Matter
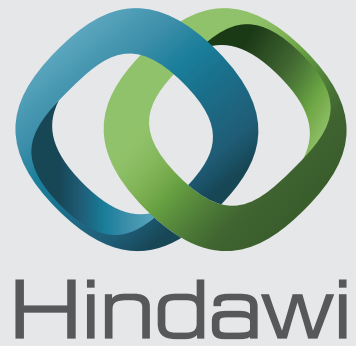

Submit your manuscripts at

http://www.hindawi.com
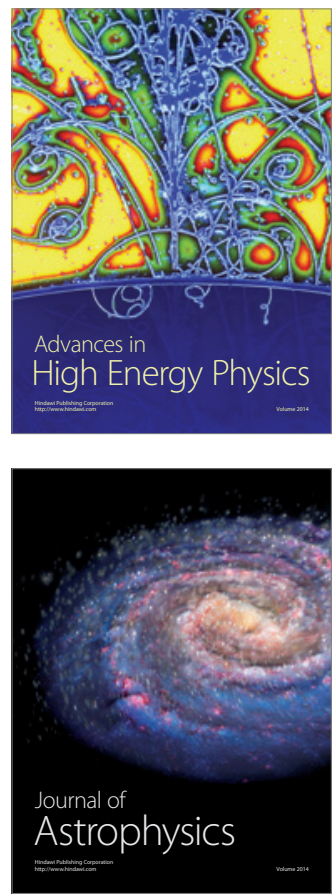
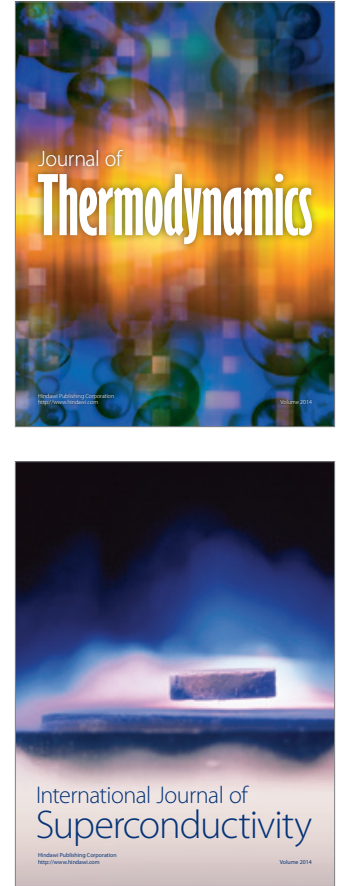
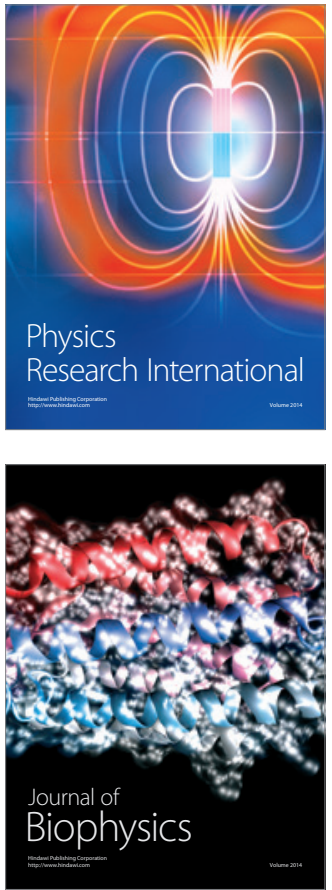
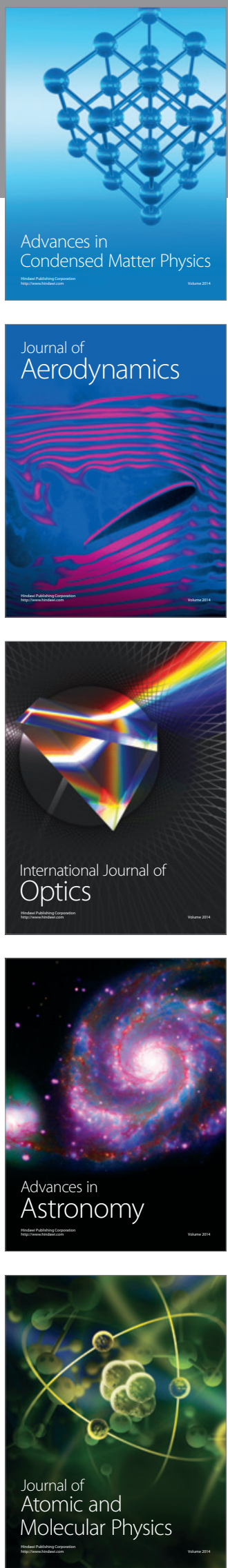